\title{
Congenital aplasia of the optic chiasm and esophageal atresia: a case report
}

\author{
Stefano Pensiero ${ }^{1}$, Paolo Cecchini ${ }^{2}$, Paola Michieletto ${ }^{2}$, Gloria Pelizzo ${ }^{2}$, Maurizio Madonia ${ }^{1}$ and Fulvio Parentin ${ }^{1,3^{*}}$
}

\begin{abstract}
Introduction: The complete absence of the chiasm (chiasmal aplasia) is a rare clinical condition. Hypoplasia of the optic nerve and congenital nystagmus are almost invariably associated characteristics. Microphthalmos or anophthalmos are common features in chiasmal aplasia, while central nervous system abnormalities are less frequent. Esophageal atresia can be isolated or syndromic. In syndromic cases, it is frequently associated with cardiac, limb, renal or vertebral malformations and anal atresia. More rarely, esophageal atresia can be part of anophthalmia-esophageal-genital syndrome, which comprises anophthalmia or microphthalmia, genital abnormalities, vertebral defects and cerebral malformations. Here, a previously unreported case of chiasmal aplasia presenting without microphthalmos and associated with esophageal atresia is described.
\end{abstract}

Case presentation: Aplasia of the optic chiasm was identified in a Caucasian Italian 8-month-old boy with esophageal atresia. An ultrasound examination carried out at 21 weeks' gestation revealed polyhydramnios. Intrauterine growth retardation, esophageal atresia and a small atrial-septal defect were subsequently detected at 28 weeks' gestation. Repair of the esophageal atresia was carried out shortly after birth. A jejunostomy was carried out at four months to facilitate enteral feeding. The child was subsequently noted to be visually inattentive and to be neurodevelopmentally delayed. Magnetic resonance imaging revealed chiasmal aplasia. No other midline brain defects were found. His karyotype was normal.

Conclusion: If achiasmia is a spectrum, our patient seems to depict the most severe form, since he appears to have an extremely severe visual impairment. This is in contrast to most of the cases described in the literature, where patients maintain good-or at least useful- visual function. To the best of our knowledge, the association of optic nerve hypoplasia, complete chiasmal aplasia, esophageal atresia and atrial-septal defect, choanal atresia, hypertelorism and psychomotor retardation has never been described before.

\section{Introduction}

Complete absence of the chiasmal structure, often associated with optic nerve aplasia, is termed chiasmal aplasia, while the term achiasmia is used to identify the abnormality of crossing fibers [1]. In fact, while in albinism the temporal retinal fibers erroneously decussate at the optic chiasm (OC), in achiasmia the majority of fibers fail to cross at the $\mathrm{OC}$ and project ipsilaterally. This condition is also termed 'non-decussating retinalfugal fiber syndrome' (NDRFFS) [2,3]. Both albino and achiasmatic anatomical and developmental abnormalities can be functionally demonstrated by means of flash

\footnotetext{
* Correspondence: parentin@burlo.trieste.it

'Ophthalmology Unit, Department of Surgery, Institute for Maternal and Child Health, Burlo Garofolo Trieste, Via dell'Istria 65/1, I-34100 Trieste, Italy Full list of author information is available at the end of the article
}

visual evoked potentials (F-VEPs) [4,5]. In achiasmia, FVEPs show a higher positive component ipsilateral to the stimulated eye, while in albinos the response is greater contralaterally $[4,1]$.

Congenital nystagmus is a consistent feature in achiasmia, as well as in certain cases of optic nerve hypoplasia $[2,6]$. Central nervous system (CNS) abnormalities, such as septo-optic dysplasia, hypopituitarism, encephalocele or corpus callosum agenesia are associated with achiasmia [1].

The complete absence of the chiasm (chiasmal aplasia) is a rarer clinical condition. Hypoplasia of the optic nerve and congenital nystagmus are almost invariably associated characteristics [6,7]. Microphthalmos or anophthalmos are common features in chiasmal aplasia, while CNS abnormalities are less frequent $[6,7]$.

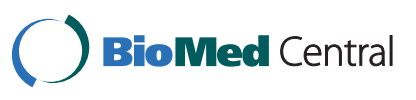


A case of chiasmal aplasia in a Caucasian baby, associated with esophageal atresia (EA), is here described.

\section{Case presentation}

Polyhydramnios was detected in a 35-year-old primigravida at 21 weeks' gestation. Esophageal atresia was diagnosed at 28 weeks' gestation on the basis of a small stomach and polyhydramnios on an ultrasound examination. Other abnormalities detected included a dilated upper esophageal pouch and an atrial-septal defect. Our patient underwent periodic therapeutic amniocentesis (1500-1900 ml of fluid per procedure) from 28 weeks' gestation for relief of polyhydramnios and to prevent premature onset of labor. A male fetus with a birth weight of $1980 \mathrm{~g}$ was delivered by caesarean section at 37 weeks' gestation. Apgar scores were six and eight at one and five minutes respectively. A clinical examination showed type III EA, right choanal atresia, atrial-septal defect, telecanthus and hypertelorism without any obvious strabismus. His mother's history was negative for familial visual anomalies and there was no evidence of maternal infection or drug abuse during pregnancy. His karyotype was normal.

Uncomplicated esophageal anastomosis with closure of the tracheo-esophageal fistula was performed on the second day of his life. Due to the persistence of suction weakness, our patient was discharged two months later with a naso-gastric tube and enteral nutrition. The baby was referred for pediatric consultation at four months of age. A failure to thrive (length and weight, head circumference on third centile) due to gastroesophageal reflux was documented. A laparoscopic gastric fundoplication was performed. A jejunostomy was also performed to allow continuation of enteral feeding.

The baby underwent a complete ophthalmic evaluation at the age of four months: our patient showed erratic eye movements and was unable to fixate on a light. Horizontal slow nystagmus was observed. His pupil reactivity to light was absent bilaterally. A slit-lamp biomicroscopy of the anterior segment of both eyes was unremarkable. Funduscopy revealed severe bilateral optic disc hypoplasia and mild tortuosity of the retinal blood vessels. His optic discs appeared greyish and oval. F-VEPs were repeatedly non-recordable bilaterally.

Brain magnetic resonance imaging (MRI) scans performed at the age of nine months showed normal eye bulb structures, absence of the $\mathrm{OC}$ and optic radiations, consistent with extreme hypoplasia or aplasia of those structures. His optic nerves were bilaterally traced only in the intraorbital portion and were of small appearance. There was no evidence of his optic nerves more posteriorly (Figure 1). His other cerebral structures were normal.
An evaluation at 10 months revealed delayed social skills and language development.

General examination at 18 months of life showed reduced stature, persistence of food aversion, and delayed social contacts and language development.

\section{Discussion}

EA can be isolated or syndromic [8]. In syndromic cases, EA is frequently associated with cardiac, limb, renal and vertebral malformations and anal atresia [8]. In our case, his karyotype was normal, so chromosomal anomalies responsible of syndromic EA (for example trisomy 21, 18, 13 and 17q21.3-q23 deletion) were not involved [8]. Other conditions frequently associated with EA include VACTERL (vertebral anomalies, anal atresia, cardiovascular anomalies, trachea-esophageal fistula, esophageal atresia, renal and/or radial anomalies and limb defects) syndrome, Feingold (oculo-digito-esophago-duodenal) syndrome and Rogers (anophthalmiaesophageal-genital or AEG) syndrome [8]. Other conditions occasionally associated with EA, and characterized by an ocular involvement, are shown in Table 1. Maternal diabetes and phenylketonuria, rarely associated with EA, were excluded due to the normality of blood tests during pregnancy. AEG syndrome comprises EA, anophthalmia or microphthalmia, genital abnormalities, vertebral defects and cerebral malformations [9]. There is evidence of a genetic mechanism for this syndrome. In a number of cases, deletion and/or mutation of the transcriptional regulator gene, Sox2, has been proved to be involved $[10,11]$. The phenotypic variability may be remarkable, ranging from anophthalmia to normal ocular development $[12,13]$. Sox 2 also seems to play a causative role in isolated anophthalmia and microphthalmia [11]. However, S Sox2 deletion or malfunction can hardly have been involved in our patient, as his eyes were fully developed and the only eye abnormality was the optic nerve hypoplasia. Moreover he presented with no genital or vertebral anomalies, while he did have a heart malformation. He did not present ear anomalies, typical of CHARGE (coloboma of the eye, heart defects, atresia of the choanae, retardation of growth, genital abnormalities, and ear abnormalities) and OAVS (oculoauriculo-vertebral spectrum) syndromes, but the first is the only syndrome presenting with choanal atresia (Table 1). Our patient could not therefore be classified in any of the syndromes associated with EA.

In syndromes associated with optic nerve and/or OC hypoaplasia, some genes responsible for the molecular mechanisms of neural routing have been related to the achiasmia spectrum. In the animal model, the lack of the transcription factor Foxd1 induces chiasmal malformation and misprojection of retinal fibers [14]. Foxd1 


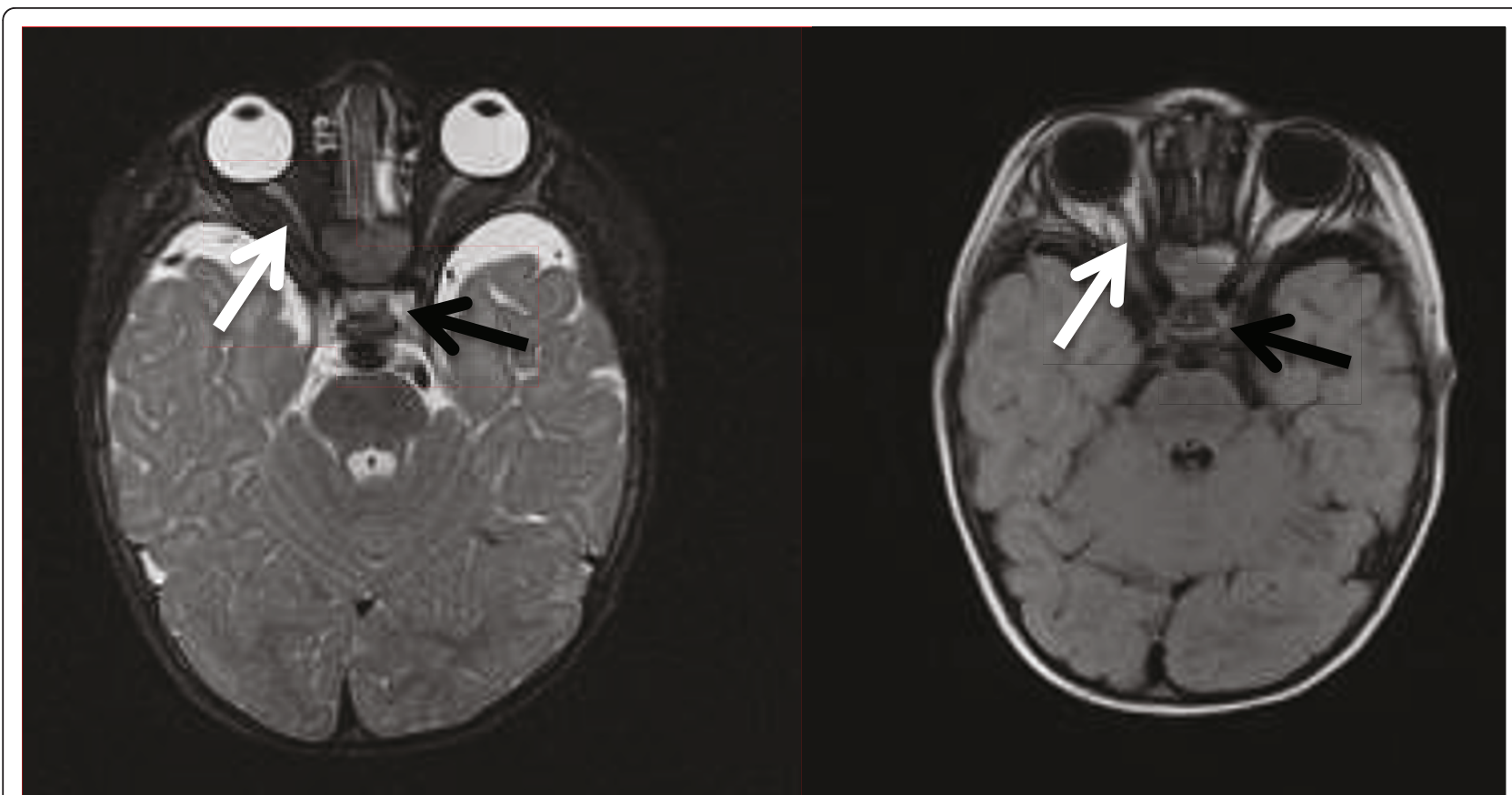

Figure 1 Axial Tx and Ty MRI scans showing complete absence of the optic chiasm (black arrow); the optic nerves can be identified only in the intraorbital portion (white arrow).

Table 1 Comparison among the clinical characteristic of our case and the syndromic form of EA

\begin{tabular}{|c|c|c|c|c|c|c|c|}
\hline Features & Our Case & AEG (Rogers) & VACTERL & Feingold & CHARGE & OAVS & Bartsocas-Papas \\
\hline Esophageal atresia & + & + & + & + & + & + & + \\
\hline Microcephaly & - & + & - & + & + & - & + \\
\hline Optic chiasm aplasia & + & - & - & - & - & - & - \\
\hline Nystagmus & + & - & - & - & - & - & - \\
\hline Optic nerve hypoplasia & + & - & - & - & - & - & - \\
\hline Eyes anomalies & - & + & - & + & + & + & + \\
\hline Optic tract aplasia & + & - & - & - & - & - & - \\
\hline Anophthalmia/microphthalmia & - & + & - & - & - & - & + \\
\hline Telecanthus/hypertelorism & + & - & - & - & - & - & + \\
\hline Cerebral malformation & - & - & - & - & + & - & + \\
\hline Heart malformation & + & - & + & + & + & + & - \\
\hline Vertebral defects/other bone anomalies & - & - & + & + & + & + & + \\
\hline Genital/renal anomalies & - & + & + & + & + & + & + \\
\hline Facial/visceral problems & - & - & - & - & - & + & + \\
\hline Visual impairment & + & - & - & - & - & - & - \\
\hline Mental retardation & + & + & - & + & + & - & - \\
\hline Facial cleft & - & - & - & - & - & - & + \\
\hline Limb anomalies & - & - & + & - & + & - & + \\
\hline Ear deformities & - & - & - & - & + & + & - \\
\hline Choanal atresia & + & - & - & - & + & - & - \\
\hline
\end{tabular}


misexpression leads to a cascade of other gene products misregulation, which interferes with the normal development of the OC and with the ratio of ipsilateral to contralateral chiasmal nerve fiber routing [14]. Foxd1 has not been described to play a role in EA and it is questionable how it could be involved in the multiple malformations observed in the present report. It seems unlikely to consider EA a merely incidental finding. Pax family genes have also been suggested to play an important role in the correct development of the OC $[15,16]$. In the murine Pax mutant model, the OC fails to develop, and retinal axons enter the ipsilateral optic tract $[15,16]$. Again, the association with EA in our case would be hard to explain.

Sami et al. [1] have suggested a classification system for patients affected by achiasmia: type A, presenting with isolated achiasmia and often nystagmus, with possible MRI evidence of a small chiasm; type B, presenting with chiasmal hypoplasia and midline defects (septooptic dysplasia); and type $C$, presenting with chiasmal hypoplasia and possible clefting disorders, encephalocele, and agenesia of the corpus callosum. In Sami's series, one patient did not fit into one of the three suggested groups. The child (a boy of six years) suffered from multiple facial, visceral and developmental problems, including EA. An MRI scan showed an isolated small chiasm. The child exhibited horizontal nystagmus. To the best of our knowledge, this is the only previous case of achiasmia in a patient affected by EA, and appears somehow similar to ours: OC aplasia, horizontal nystagmus and esophageal atresia are shared features between Sami's case and ours. However, other traits are remarkably different. The main discrepancy is the presence of the chiasm-even if hypoplasic-in the MRI scans in Sami's report, while no chiasm structure was detectable on MRI scanning and no facial abnormalities were present in our patient. The F-VEPs results and the lack of fixation and of pupil reactivity to light suggest a severe visual impairment in this child, unlike the Sami case, who exhibited fairly good visual function. Moreover our child showed an atrial septal defect and choanal atresia, which was not been described in the Sami case.

One could speculate that these two cases may have the same etiology with different phenotypes. The rarity of this condition and the relevant differences between the two cases, however, suggest great caution in attempting to group them in a single clinical entity.

If achiasmia is a spectrum, our child seemed to depict the most severe form, since he appeared to have an extremely severe visual impairment, in contrast to most of the cases described in literature that maintain a goodor at least useful-visual function. The lack of fixation and reactivity to light or structured stimuli and the presence of roving eye movements were highly suggestive of poor or no residual visual function. The lack of F-VEP response-which is very unusual in achiasmia-confirmed the OC aplasia suggested by MRI findings; moreover we could consider the OC aplasia of our patient to be secondary to a primary bilateral severe optic nerve hypoplasia. Pomeranz [17] described an 18-month-old boy with bilateral optic nerve hypoplasia and OC not identifiable at the MRI who showed profoundly abnormal F-VEP in his right eye. However, left eye stimulation demonstrated a typical VEP occipital asymmetry of the response, consistent with NDRFFS. VEP results showed the presence of a hypoplasic and not aplasic OC (OC was not detectable using MRI scans), with the characteristics of achiasmia. On MRI scans no other major brain abnormalities were detected. In contrast to our case, no other visceral malformations were noticed, and the baby showed a good visual interaction with the environment.

Finally, OC aplasia has often been described in association with other major CNS abnormalities and unilateral or bilateral anophthalmos or microphthalmos was always present. This is in contrast to our case. The genes that are involved in EA and in achiasmia are all located in different chromosomes, so it is difficult to predict simultaneous involvement of multiple genes. In fact Pax is located in chromosome 11p13, Foxd11 on 5q12-13, Mycn (producing Feingold syndrome) on 2p24.1, Chd7 (CHARGE) on 8q12.2 and Sox2 (AEG) on $3 q 26$. For this reason the syndrome here described, like VACTERL and OAVS, is probably to be considered of malformative origin.

\section{Conclusion}

We believe that our child does not fit into one of the previously reported achiasmia or OC aplasia types or reports. To the best of our knowledge, the association of optic nerve hypoplasia, chiasmal and optic tracts aplasia (confirmed by VEP and light pupil reactivity absence), with telecanthus and/or hypertelorism, esophageal atresia, atrial septal defect, choanal atresia, and developmental and language delays has never been described before.

\section{Consent}

Written informed consent was obtained from the parents of the patient for publication of this case report and any accompanying images. A copy of the written consent is available for review by the Editor-in-Chief of this journal.

\section{Abbreviations}

AEG: anophthalmia-esophageal-genital; CNS: central nervous system; EA: esophageal atresia; F-VEPs: flash visual evoked potentials; MRI: magnetic resonance imaging; NDRFFS: non-decussating retinal fugal fiber syndrome; OC: optic chiasm. 


\section{Author details}

'Ophthalmology Unit, Department of Surgery, Institute for Maternal and Child Health, Burlo Garofolo Trieste, Via dell'Istria 65/1, I-34100 Trieste, Italy. ${ }^{2}$ IRCCS E Medea, Via Cialdini 5, I-33037 Pasian di Prato (UD), Italy. ${ }^{3}$ Paediatric Surgery Unit, Department of Surgery, Institute for Maternal and Child Health, Burlo Garofolo Trieste, Via dell'Istria 65/1, I-34100 Trieste, Italy.

\section{Authors' contributions}

SP was a major contributor in writing the manuscript. GP performed surgical intervention.

PC and PM performed clinical and instrumental examinations. MM and FP made a review of the literature and were involved in the diagnosis and management of the patient. All authors have read and approved the final manuscript.

\section{Competing interests}

The authors declare that they have no competing interests.

Received: 13 August 2010 Accepted: 1 August 2011

Published: 1 August 2011

\section{References}

1. Sami DA, Saunders D, Thompson DA, Russell-Eggitt IM, Nischal KK, Jeffrey G, Dattani M, Clement RA, Liasis A, Taylor DS: The achiasmia spectrum: congenitally reduced chiasmal decussation. Br J Ophthalmol 2005, 89(10):1311-1317.

2. Korff CM, Apkarian P, Bour L, Meuli R, Verrey JD, Roulet Perez E: Isolated absence of optic chiasm revealed by congenital nystagmus, MRI and VEPs. Neuropediatrics 2003, 34(4):219-223.

3. Apkarian $P$, Bour $L J$, Barth $P G$, Wenniger-Prick L, Verbeeten B Jr: A unique achiasmatic anomaly detected in non-albinos with misrouted retinalfugal projections. Eur J Neurosci 1994, 6(3):501-507.

4. Thompson DA, Kriss A, Chong K: Visual-evoked potential evidence of chiasmal hypoplasia. Ophthalmology 1999, 106(12):2354-2361.

5. Leitch RJ, Thompson D, Harris CM, Chong K, Russell-Eggitt I, Kriss A: Achiasmia in a case of midline craniofacial cleft with seesaw nystagmus. Br J Ophthalmol 1996, 80(11):1023-1024.

6. Biega TJ, Khademian ZP, Vezina : Isolated absence of the optic chiasm: a rare cause of congenital nystagmus. Am J Neuroradiol 2007, 28(2):392-393.

7. Sanjari MS, Ghasemi Falavarjani K, Parvaresh MM, Kharazi HH, Kashkooli MB: Bilateral aplasia of the optic nerve, chiasm, and tracts in an otherwise healthy infant. Br J Ophthalmol 2006, 90(4):513-514.

8. Geneviève D, de Pontual L, Amiel J, Sarnacki S, Lyonnet S: An overview of isolated and syndromic oesophageal atresia. Clin Genet 2007, 71(5):392-399.

9. Shah $D$, Jones $R$, Porter $H$, Turnpenny P: Bilateral microphthalmia, esophageal atresia, and cryptorchidism: the anophthalmia-esophagealgenital syndrome. Am J Med Genet 1997, 70(2):171-173.

10. Hill CJ, Pilz DT, Harper PS, Castle B, Williams TH: Anophthalmia-esophagealgenital syndrome: a further case to define the phenotype. Am J Med Genet 2005, 132(1):57-59.

11. Zhou J, Kherani F, Bardakjian TM, Katowitz J, Hughes N, Schimmenti LA, Schneider A, Young TL: Identification of novel mutations and sequence variants in the SOX2 and CHX10 genes in patients with anophthalmia/ microphthalmia. Mol Vis 2008, 14:583-592.

12. Chassaing N, Gilbert-Dussardier B, Nicot F, Fermeaux V, Encha-Razavi F, Fiorenza M, Toutain A, Calvas P: Germinal mosaicism and familial recurrence of a SOX2 mutation with highly variable phenotypic expression extending from AEG syndrome to absence of ocular involvement. Am J Med Genet 2007, 143(3):289-91.

13. Zenteno JC, Perez-Cano HJ, Aguinaga M: Anophthalmia-esophageal atresia syndrome caused by an SOX2 gene deletion in monozygotic twin brothers with markedly discordant phenotypes. Am J Med Genet 2006, 140(18):1899-1903.

14. Herrera E, Marcus R, Li S, Williams SE, Erskine L, Lai E, Mason C: Foxd1 is required for proper formation of the optic chiasm. Development 2004, 131(22):5727-5739.

15. Alvarez-Bolado G, Schwarz M, Gruss P: Pax-2 in the chiasm. Cell Tissue Res 1997, 290(2):197-200
16. Torres M, Gómez-Pardo E, Gruss P: Pax2 contributes to inner ear patterning and optic nerve trajectory. Development 1996, 122(11):3381-3391.

17. Pomeranz HD, Agadzi AK, Ekesten B: Achiasmia and unilateral optic nerve hypoplasia in an otherwise healthy infant. Acta Ophthalmol Scand 2006, 84(1):140-144.

doi:10.1186/1752-1947-5-335

Cite this article as: Pensiero et al:: Congenital aplasia of the optic chiasm and esophageal atresia: a case report. Journal of Medical Case Reports 2011 5:335.

\section{Submit your next manuscript to BioMed Central and take full advantage of:}

- Convenient online submission

- Thorough peer review

- No space constraints or color figure charges

- Immediate publication on acceptance

- Inclusion in PubMed, CAS, Scopus and Google Scholar

- Research which is freely available for redistribution

Submit your manuscript at www.biomedcentral.com/submit
Ciomed Central 